\title{
Building Research Infrastructure in Community Health Centers: A Community Health Applied Research Network (CHARN) Report
}

\author{
Sonja Likumahuwa, MID, MPH, Hui Song, MPH, MS, Robbie Singal, DrPH, \\ Rosy Chang Weir, PhD, Heidi Crane, MD, MPH, John Muench, MD, MPH, \\ Shao-Chee Sim, PhD, and Jennifer E. DeVoe, MD, DPhil
}

This article introduces the Community Health Applied Research Network (CHARN), a practice-based research network of community health centers (CHCs). Established by the Health Resources and Services Administration in 2010, CHARN is a network of 4 community research nodes, each with multiple affiliated CHCs and an academic center. The four nodes (18 individual CHCs and 4 academic partners in 9 states) are supported by a data coordinating center. Here we provide case studies detailing how CHARN is building research infrastructure and capacity in CHCs, with a particular focus on how community practice-academic partnerships were facilitated by the CHARN structure. The examples provided by the CHARN nodes include many of the building blocks of research capacity: communication capacity and "matchmaking" between providers and researchers; technology transfer; research methods tailored to community practice settings; and community institutional review board infrastructure to enable community oversight. We draw lessons learned from these case studies that we hope will serve as examples for other networks, with special relevance for community-based networks seeking to build research infrastructure in primary care settings. (J Am Board Fam Med 2013;26:579-587.)

Keywords: Community Health Centers, Community Health Networks, Practice-based Research Network

Community health centers (CHCs) are increasingly interested in and recruited to participate in research but may lack infrastructure to meaningfully engage as research partners. ${ }^{1,2}$ Academic

This article was externally peer reviewed.

Submitted 14 January 2013; revised 9 May 2013; accepted 14 May 2013.

From the Department of Family Medicine, Oregon Health \& Science University, Portland (SL, JM, JED); the OCHIN Safety Net Practice-Based Research Network, Portland, OR (SL, JM, JED); the Association of Asian Pacific Community Health Organizations, Oakland, CA (HS, RCW, S-CS); The Fenway Institute at Fenway Health, Boston, MA (RS, HC); the Department of Medicine, University of Washington, Seattle (HC); and the Charles B. Wang Community Health Center, New York, NY (S-CS).

Funding: This study was supported by grant no. UB2HA20235 from the Health Resources and Services Administration. Additional support for the project was provided by the Department of Family Medicine, Oregon Health \& Science University, Portland.

Conflict of interest: none declared.

Corresponding author: Sonja Likumahuwa, MID, MPH, Oregon Health \& Science University, Department of Family Medicine, 3181 SW Sam Jackson Park Rd., Mailcode: FM, Portland, OR 97239 (E-mail: likumahu@ohsu.edu). health researchers are also becoming more aware of the potential for partnering with CHCs to conduct relevant primary care and patient-centered research but may not know how to build strong research partnerships. The extensive experience and literature from non-medical community-academic partnerships and community-based participatory research methods describes how these partnerships are formed and help overcome community academic barriers. ${ }^{3-10}$ Practice-based research networks (PBRNs) have begun learning from these experiences to inform strong health research partnerships. ${ }^{6}$ Seifer and colleagues ${ }^{2}$ outlined 5 important infrastructure components necessary to support successful research processes and outcomes: (1) a community-academic relationship, (2) relevant policies and procedures, (3) financial resources, (4) human resources, and (5) "hard" infrastructure (e.g., hardware such as computers, research software and equipment, and databases). Many efforts have been made nationally to build infrastructure 
Figure 1. Map of sites participating in the Community Health Applied Research Network.

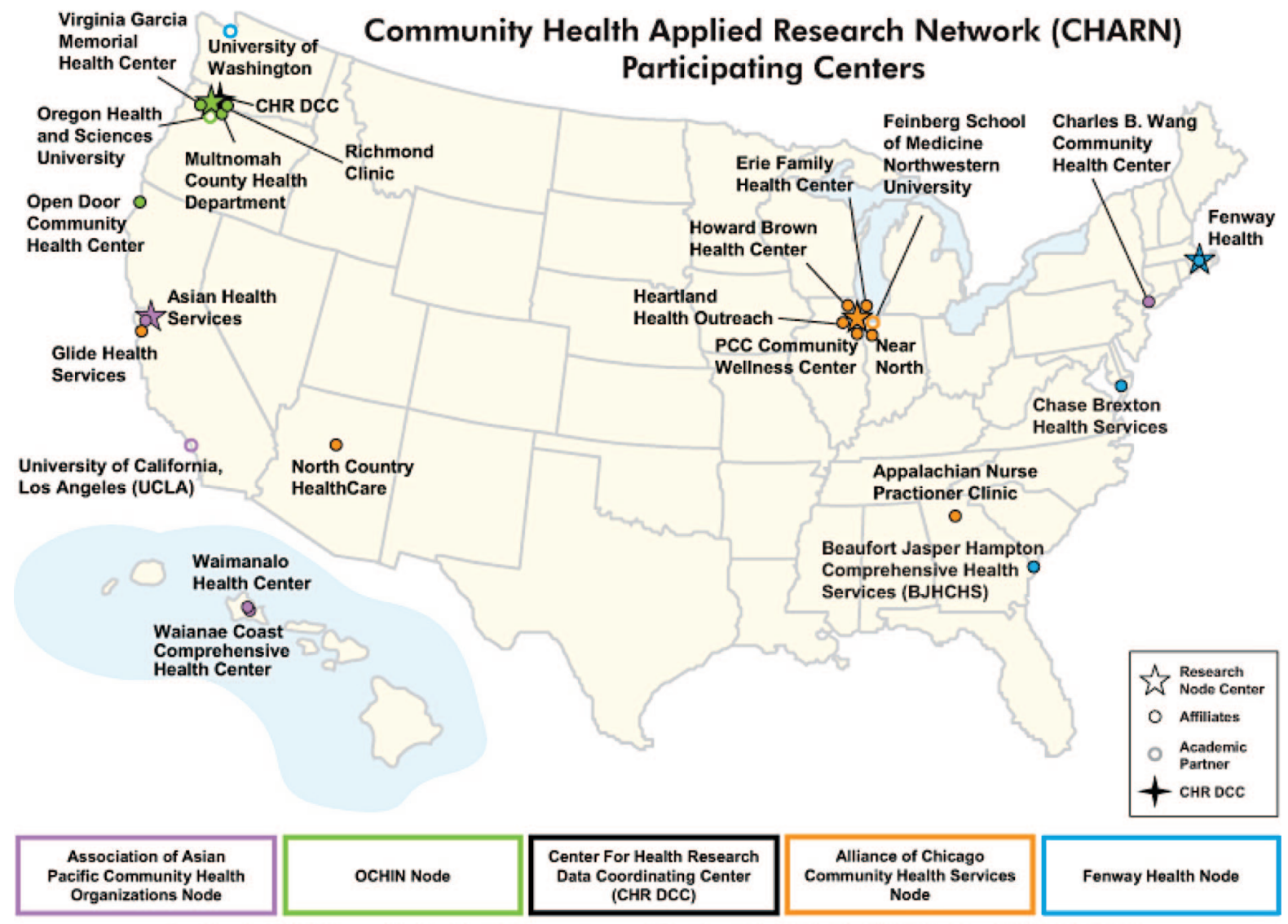

Funding for CHARN provided by Health Resources and Services Administration (HRSA); Grant \# UB3HA20236

and capacity. However, much work remains to build infrastructure that enables trust-based, equitable, collaborative research that furthers the goal of supporting the partnership that brings together clinicians' practice-based clinical questions and academic researchers' research skills.

This article introduces the Community Health Applied Research Network (CHARN), which is a network of 4 primary care PBRNs, each with a membership of CHCs. ${ }^{11}$ CHARN offers opportunities to bridge clinical practice and academic environments to improve research infrastructure and capacity. Here we present brief case studies from 3 of the 4 research nodes, highlighting some of the tangible capacity built through academic-practice partnerships fostered through the CHARN.

Established in 2010 through a grant from the Health Resources and Services Administration, CHARN consists of 4 research "nodes" and a coordinating center. The research nodes are the
Association of Asian Pacific Community Health Organizations (AAPCHO), Alliance of Chicago Community Health Services, Fenway Health, and OCHIN, Inc. Each node is a PBRN of CHCs, of which a subset participates in CHARN. The map in Figure 1 shows the CHARN CHC and academic affiliate sites across 9 states. One of the main goals of CHARN is to further community-academic partnerships for health research; therefore, each research node consists of a number of CHCs (ranging from 3 to 7) and an academic affiliate institution. The data coordinating center is part of the Kaiser Permanente Center for Health Research in Portland, Oregon, which supports scientists and their research projects by providing information technology and expertise in development, implementation, and dissemination relating to multicenter data resources and research studies. Table 1 presents the CHARN nodes, basic demographics of the CHARN, and CHARN's active research projects. 
Table 1. Characteristics of Members of the Community Health Applied Research Network (CHARN)

\begin{tabular}{|c|c|}
\hline CHARN Research Node Center & Participating CHCs and Locations \\
\hline $\begin{array}{l}\text { Association of Asian Pacific Community Health } \\
\text { Organizations (AAPCHO) }\end{array}$ & $\begin{array}{l}\text { Asian Health Services Community Health Center, Oakland, CA } \\
\text { Charles B. Wang Community Health Center, New York, NY } \\
\text { Waianae Coast Comprehensive Health Center, Waianae, HI } \\
\text { Waimanalo Health Center, Waimanalo, HI } \\
\text { University of California, Los Angeles (Academic Affiliate) }\end{array}$ \\
\hline $\begin{array}{l}\text { Alliance of Chicago Community Health } \\
\text { Services }\end{array}$ & $\begin{array}{l}\text { Erie Family Health Center, Inc., Chicago, IL } \\
\text { GLIDE Health Services, San Francisco, CA } \\
\text { Heartland Health Outreach, Chicago, IL } \\
\text { Howard Brown Health Center, Chicago, IL } \\
\text { Near North Community Health Center, Chicago, IL } \\
\text { North Country Health Care, Chicago, IL } \\
\text { PCC Community Wellness, Chicago, IL } \\
\text { Northwestern University, Chicago, IL (Academic Affiliate) }\end{array}$ \\
\hline Fenway Health & $\begin{array}{l}\text { Chase-Brexton Health Services, Baltimore, MD } \\
\text { Beaufort-Jasper-Hampton Comprehensive Health Services, Ridgeland, SC } \\
\text { Fenway Health, Boston, MA } \\
\text { University of Washington, Seattle, WA (Academic Affiliate) }\end{array}$ \\
\hline OCHIN, Inc. & $\begin{array}{l}\text { OHSU Richmond Family Health Center, Portland, OR } \\
\text { Multnomah County Health Department, Portland, OR } \\
\text { Virginia Garcia Memorial Health Center, Hillsboro, OR } \\
\text { Open Door Community Health Center, Eureka, CA } \\
\text { Oregon Health \& Science University, Portland, OR (Academic Affiliate) }\end{array}$ \\
\hline Data Coordinating Center & Center for Health Research, Kaiser Permanente, Portland, OR \\
\hline CHARN active patients (n) & 519,636 \\
\hline \multicolumn{2}{|l|}{ CHARN languages (\%) } \\
\hline English & 55.6 \\
\hline Spanish & 17.9 \\
\hline Cantonese & 5.1 \\
\hline Mandarin & 2.9 \\
\hline Other* & $<1$ \\
\hline \multicolumn{2}{|l|}{ CHARN race/ethnicity (\%) } \\
\hline White & 60.5 \\
\hline Black & 18.3 \\
\hline Asian/Native Hawaiian/Pacific Islander & 17.5 \\
\hline American Indian/Alaska Native & 0.9 \\
\hline Multiracial & 1.0 \\
\hline Other* & 5.2 \\
\hline No race indicated & 11.0 \\
\hline Active research projects supported by CHARN & $\begin{array}{l}\text { 1. Evaluating the effectiveness of enabling services for improving } \\
\text { health at community health centers nationwide } \\
\text { 2. Electronic patient-reported outcomes (ePRO) } \\
\text { 3. Improving colorectal cancer screening } \\
\text { 4. Identifying high risk cardiovascular disease patients using } \\
\text { electronic health record data } \\
\text { 5. HIV testing in CHARN-affiliated community health centers } \\
\text { 6. Characterizing the diabetes population in the CHARN } \\
\text { 7. Impact of meaningful use regulations on smoking counseling in } \\
\text { community health centers }\end{array}$ \\
\hline
\end{tabular}

*Other includes Russian, Vietnamese, Somali, Korean, Cambodian, and Arabic. $\mathrm{CHC}$, community Health Center; HIV, human immunodeficiency virus. 
The goals of CHARN are to:

- Foster practice-based collaboration among personnel, practitioners, and researchers at various clinics and centers;

- Create infrastructure for pooling patient data across different sites;

- Train CHC personnel in research methods and protocols;

- Develop and conduct study protocols;

- Expand the research agenda via additional funding; and

- Develop improved approaches for transferring research findings into practice.

CHARN's unique structure is designed to facilitate communication between community-based practitioners and academic researchers. The CHARN Steering Committee (SC) is a 22 -member body that sets research priorities, oversees research projects using CHARN data, and reinforces a unified vision through which to carry out CHARN objectives. The membership of the SC consists of a principal investigator (PI) from each of the 4 research nodes, 3 representatives from clinical and academic affiliates designated by each research node, 3 data coordinating center representatives (the PI and 2 other representatives selected by the PI), and 3 experts nominated by the Health Resources and Services Administration. Each clinical affiliate has a "site lead" who serves as a conduit of information between the SC and the clinic site. In addition to the SC, clinicians and researchers serve together on a research planning committee and 7 project-specific workgroups.

CHARN was developed out of a desire to engage $\mathrm{CHCs}$ as partners in research and to empower and build their research capacity. CHCs are ideal partners with whom to conduct patient-centered outcomes research because they engage in quality improvement and evaluation with a mission to provide efficient and effective care that advances health and reduces disparities. They also integrate essential enabling services (e.g., interpretation, eligibility assistance) that facilitate access to care and are increasingly using innovations in electronic health records (EHRs) and health information technology. ${ }^{12}$ More important, CHCs are rooted in the communities they serve and are governed by boards of directors, with a majority representing consumers. By partnering with CHCs, research- ers can engage with and hear directly from patients, providers, caregivers, and other key stakeholders regarding health care services delivered through the safety net. Little is known about the populations served by $\mathrm{CHCs}$, including those populations' responses to illness and understanding of health and health care needs, the value of providing comprehensive care to a complex population, and responding appropriately to the medical and psychosocial challenges faced by their underserved populations. ${ }^{1}$ Research provides CHCs an opportunity to realize and interpret their own evidence and integrate this evidence into practice to improve health care delivery and influence public policy. Only with rigorous research can CHCs provide critical evidence about the effectiveness of their services to their unique patient base. ${ }^{1}$

Too often, however, research fails to include $\mathrm{CHCs}$ as health care innovators or a vital part of the safety net. CHCs have the potential to promote health, prevent disease, address health disparities, and translate research findings to their medically underserved patient populations. In addition, CHCs will increasingly be required to produce evidence of high-quality practices to gain and sustain increased pay for better performance on quality measures. The many capable and dedicated personnel in CHCs may lack the skills, protected time, and resources required to conduct the research needed for quality improvement in health care practice and develop and evaluate the programs in their communities. They need support and funding to successfully engage in patientcentered outcomes research in the CHC setting. CHARN conducted an online survey in 2011 to assess research needs and capacity of CHCs in our network. We found that the most commonly reported challenges to engaging in research were lack of staff time (90\%), concern about lost productivity (80\%), and lack of funding opportunities (70\%) (manuscript in preparation). Overcoming these challenges will not only generate important knowledge about best practices implemented at CHCs to address health disparities and improve patient care but also help CHCs to build their own capacity and the necessary infrastructure to successfully engage in patient-centered outcomes research and evaluation enterprises. CHCs can also facilitate the dissemination of findings from the perspective of a community participant. Research study findings are often not disseminated to the community in a way that is mean- 
ingful and useful. ${ }^{13}$ CHCs have invaluable expertise related to the most appropriate methods to reach local communities given the longstanding relationships of trust they have developed with their respective communities. Although the 4 research nodes comprising CHARN were already working to engage and build capacity among their members, the CHARN funding has enabled further innovative linkages between further academic and community partners.

\section{Case Studies in the Development of CHC Research Infrastructure}

Through CHARN's multidirectional communication structure, we solicited from CHARN CHC site leads and their academic partners concrete examples of improvements in research infrastructure and capacity. We asked CHCs to focus on how academic-practice partnerships were facilitated by the CHARN structure.

\section{OCHIN: Enbancing a PBRN's Ability to Conduct Clinician-Driven Research}

Originally known as the Oregon Community Health Information Network and shortened to OCHIN as other states joined, OCHIN is a collaborative, member-based organization that is nationally recognized for its innovative use of health information technology to improve the integration and delivery of health care services. The OCHIN PBRN was founded in 2007 and includes all 63 OCHIN members (a wide variety of practices with an emphasis on safety net clinics, small practices, critical access, and rural hospitals). Of these, 4 chose to participate in CHARN: the Open Door Community Health Center (Humboldt and Del Norte counties, California); Virginia Garcia Memorial Health Center (Washington and Yamhill counties, Oregon); Multnomah County Health Department (Portland, Oregon); and Richmond Family Medicine Clinic (Portland, OR). The CHARN academic affiliate institution is Oregon Health \& Science University (Portland, OR).

The OCHIN PBRN has struggled to facilitate clinician-driven research. ${ }^{14}$ OCHIN invested a large amount of its CHARN funding to augment its PBRN staff and clinic champions for research at the clinics most involved with the PBRN. The tangible fruit of that investment is a significantly stronger capacity for provider-researcher communication and "matchmaking" between providers and researchers, which was achieved in 2 ways. First, they increased the PBRN staff, fully funding a PBRN coordinator and a part-time community research liaison. These roles have helped develop personal relationships with both clinicians and researchers. Second, clinic champions at the 4 CHARN CHCs are compensated for a small amount of effort to participate more fully in the PBRN and be research liaisons at their clinics.

As an example, in 2010 a study entitled "Medication Assisted Treatment for Addictive Disorders within Community Health Centers: Patient Characteristics and Comorbidities" examined similarities and differences in patients with a diagnosis of opioid dependence based on their treatment regimen (one group of patients was being treated with buprenorphine while the other group was being treated with methadone). The study was initiated by a clinical researcher at Oregon Health \& Science University (the CHARN academic affiliate) who contacted two PBRN member providers based on their clinical expertise and her knowledge and experience with the PBRN network. One of these member providers was the CHARN clinic champion at his clinic. The investigator and one provider then engaged another physician, both of whom were not previously involved with research. Collaboratively, they developed a project to address clinical questions they had encountered in practice. The partners worked together to select necessary data, guide analysis, and interpret findings. The study, now complete, characterized safety net patients dependent on opioids as low-income, with multiple comorbidities, high service utilization rates, and higher rates of homelessness. The provider-researcher team reported remarkable learning and greater research impact as a result of their collaboration. They continue to collaborate and are developing two research proposals for both federal and foundation funding. As they have developed their knowledge and skills related to using OCHIN data for research, they are also engaging other physicians, nurse practitioners, and residents in the next phases of their research agenda. The study's success was facilitated by the PBRN staff and clinic champion.

A second OCHIN CHARN affiliate, Open Door Community Health Centers, created a research director position for the physician who is its CHARN site lead. Through the CHARN invest- 
ment, this research director was able to take time from his clinical duties to become a leader in the PBRN (vice-chair of the SC). He has been active in developing ideas for research that will use the CHARN data warehouse, designing the next phase of the data warehouse, and developing his own research program in collaboration with CHARN academic researchers. The academic researchers provide input on study design, help determine the parameters for data needs, and assist with analysis.

Through the academic-practice partnership funded and enabled by CHARN, the OCHIN PBRN is realizing the goal of facilitating provider-driven research. PBRN members led the development of 4 research project proposals in CHARN and, because of the additional staff, average PBRN attendance has grown 60\% over 2 years, which brings even more providers and researchers together. Providers often identify questions in clinical practice that need to be better understood. With the help of the PBRN and the CHARN infrastructure linked to academic partners, it has become possible to turn some of these clinical questions into research questions-and eventually into answers.

\section{Fenway: Clinic-Centered Research Methods and Implementation}

The mission of Fenway Health is to enhance the well-being of the lesbian, gay, bisexual, and transgender community and all people in the surrounding neighborhoods through access to the highest quality health care, education, research, and advocacy. The CHARN Fenway Health node comprises 3 CHCs, including Chase-Brexton Health Services (5 sites in Maryland), Beaufort-Jasper-Hampton Comprehensive Health Services (8 sites in South Carolina), and Fenway Health (4 sites in Boston, MA), and their academic affiliate, the University of Washington in Seattle.

Advances in the computer-assisted collection of patient-reported data enables the rapid collection of patient behaviors, symptoms, and preferences at the point of care and the immediate integration of that information into the patient-provider encounter via the EHR. This facilitates improved patient-provider communication and better clinical care and provides quality data that often is not available for clinic research. With additional support from the Office of Behavioral and Social Sciences Research of the National Institutes of Health, CHARN supports an electronic patient- reported outcomes (ePRO) project that has introduced, adapted, and tested the collection and integration of patient-reported data captured electronically using touch-screen tablets in routine care in several CHCs across the United States. This project demonstrated CHARN support for technology transfer to CHCs and specifically uses a state-of-the-art platform to conduct computer-assisted self-administered interviews to collect patient data about depression, substance use, risky sexual behavior, and medication adherence at the time of routine clinical care visits at $3 \mathrm{CHARN}$ CHCs.

Through CHARN's committee structure, academic researchers worked with community providers to respond to the technological and feasibility challenges that were associated with implementing the ePRO project at all 3 participating CHCs. Clinical staff identified 3 main priority areas: (1) keep patients' burden low; (2) integrate data collection with clinical workflow; and (3) provide realtime data that are available to providers at the time of the appointment. An additional challenge was working with EHR and other computing systems that vary between CHCs. Implementation challenges and successes were discussed during monthly calls with the CHARN Fenway node.

A number of steps were needed to overcome the barriers to implementing ePROs. First and foremost, provider and staff support was necessary. CHARN infrastructure funds were used to cover part of the salary of a research assistant at each $\mathrm{CHC}$. This person worked closely with $\mathrm{CHC}$ staff to implement the ePRO project. Before implementation, all $3 \mathrm{CHCs}$ held medical team meetings to present the benefits associated with using ePRO tablets to collect data. The goal of these meetings was to demonstrate the potential for ePRO to reduce, not increase, their workload and improve clinical care. Data on rates of missed cases of depression, substance use, and inadequate medication adherence that was detected by ePRO data but not identified in the EHR from one pilot site were presented. The providers also were included in discussions to identify health priorities before selecting final ePRO domains and contribute to how the surveys should be administered in their clinical setting.

Integration was tailored to each clinical site based on patterns of clinical flow and where patients spend time waiting. For example, clinics with 
a provider-centric flow, where an individual provider sees patients in the same 1 or 2 examination rooms, required a much different integration pattern than clinics with less common flow patterns, such as patient-centric flow, where providers are not assigned their own rooms and patients are put in an examination room and nurses, providers, and case managers all see the patient in the examination room without the patient returning to the waiting room. With the first pattern, completing the ePRO assessments in the waiting room resulted in more seamless integration, whereas in the second pattern, completion of the assessment worked better in the examination rooms. By tailoring integration to individual clinics, this facilitated more seamless integration and higher completion rates before the patient seeing the provider.

The ePRO project seeks to make care more patient-centered and facilitate more meaningful engagement between providers and patients by determining patients' preferences, risk behaviors, and symptoms and making those data available to the provider during the encounter to improve clinical care. The project has built tangible capacity at the participating CHCs. First, ePRO technology remains in place after the project and becomes standard of care. Second, clinics have the ability to add other instruments/data collection tools as needed for clinical care and/or clinical research goals. Third, participating clinics share knowledge about implementation across participating sites, which improves understanding of real-world implementation challenges and establishes communication between academic researchers who developed the ePRO platform and CHC clinicians who use the resulting data for clinical care and research.

\section{AAPCHO: Building a Community Institutional Review Board}

AAPCHO was founded in 1987 and is a CHCgoverned network representing 29 community health organizations. These are primarily federally qualified health centers providing comprehensive, culturally and linguistically appropriate health care services to medically underserved Asian American, Native Hawaiian, and other $\mathrm{Pa}$ cific Islander (AA\&NHOPI) populations. Their member health centers are located across the country, serving more than 450,000 patients annually, with a mission to improve health status and access of AA\&NHOPI populations. The 4 AAPCHO CHCs participating in CHARN are Asian Health Services Community Health Center (Oakland, CA), Charles B. Wang Community Health Center (New York, NY), Waianae Coast Comprehensive Health Center (Waianae, HI), and Waimanalo Health Center (Waimanalo, HI). AAPCHO's academic partner in CHARN is the University of California, Los Angeles.

With more AAPCHO member CHCs participating in research, human subjects protection in community research has become a growing concern. CHCs and community based organizations, including AAPCHO, traditionally rely heavily on university institutional review boards (IRBs) to approve, monitor, and review research involving human subjects. IRB approval is required before undertaking research (it is mandated by the federal government) and thus can influence the access to federal research funding for community entities. In some cases, CHCs have had to collaborate with academic researchers to receive federal funding in part because of the need for IRB approval. However, university IRBs are often slow, traditionally lack community research experience and expertise, and may not fully understand the community interests and cultural norms or the risks of conducting research with communities having specific needs, such as the AA\&NHOPI community. In addition, being dependent on university IRBs prohibits AAPCHO and its member CHCs from independently applying for research grants to ensure that the research integrates the mission and vision of CHCs and community research criteria. ${ }^{15}$ These factors led to a direct request from AAPCHO members asking AAPCHO to develop a community IRB consistent with AAPCHO's communitybased participatory research principles and cultural values. ${ }^{16}$

After obtaining approval from its Board of Directors, a governing entity composed of executives of AAPCHO's CHC member organizations, AAP$\mathrm{CHO}$, in collaboration with its academic partner and CHC members in CHARN, started the process of developing its own community IRB that ensures that AAPCHO or member-initiated research is relevant to AAPCHO members or their communities and conforms to high standards of research integrity. CHARN has provided a useful platform for information/resource sharing in the process of IRB development. During a site visit facilitated by CHARN, staff from Howard Brown 
Health Center, a member CHC of the Alliance node, shared tips and lessons learned from their years of experience administering a community IRB that serves special populations. In addition, AAPCHO staff received much useful information from being part of the CHARN IRB committee. With templates and technical assistance from fellow CHARN organizations that have IRBs, policies and procedures have been developed to guide AAPCHO's community IRB. The IRB comprises seasoned academic and community researchers (eg, $\mathrm{CHC}$ research department directors) as well as $\mathrm{CHC}$ providers and community members who may be less involved in research but are more grounded in working with underserved communities in daily practice. Because the issue arose from the $4 \mathrm{CHCs}$, each has committed to having at least one staff member serve on the IRB. All reviewers have experience with community research and are recruited largely from participating members of CHARN, AAPCHO's existing National Research Advisory Committee, and other AAPCHO member CHCs that are not part of CHARN. All reviewers also have completed standard human subjects training and training from AAPCHO that covers considerations for conducting research review for AAPCHO and its members. Part of the CHARN funding covers the start-up costs of the development of the community IRB. A dedicated staff member will serve as the IRB coordinator to coordinate all applications and schedule review meetings. ${ }^{17}$ Bimonthly review meetings are held to conduct IRB reviews; the first meeting occurred in February 2013.

Having its own community IRB enables AAP$\mathrm{CHO}$ and its member CHCs to become truly equal partners in research, making joint decisions about what should be studied, how it should be studied, who should own the data, and how findings should be interpreted and disseminated. It shifts the control of research to AAPCHO and its member CHCs, allowing CHCs to conduct work that best fits their missions and values and is culturally and linguistically appropriate for the communities they serve. In addition, community participation through a community IRB gives a voice to underrepresented communities in research and enhances the real-world applicability of research interventions, increasing the chances of $\mathrm{CHC}$ and community buy-in and future dissemination. Furthermore, the community IRB engages and educates community programs and partners on the importance of having oversight of ethical treatment of human subjects in research, particularly for the vulnerable populations served by the CHCs. Overall, having a community IRB housed at AAPCHO provides an important opportunity to increase the research capacity and sustainability of AAPCHO member CHCs. It is also an important step for AAPCHO and its member CHCs to apply for/conduct research projects independent of academic institutions, thus leveraging more research resources for the CHCs and the rest of the community.

\section{Discussion and Conclusion}

These case studies demonstrate the considerable flexibility in how CHARN nodes approached research capacity-building. In each case, some academic-community barriers were overcome and community clinics gained research capacity that will provide a strong foundation for research that brings together the strengths of both academics and communities. CHARN has successfully supplied and/or enhanced among the CHARN CHCs the necessary infrastructure components described by Seifer et al, ${ }^{2}$ and the examples provided here demonstrate important advances in the development of research capacity. This was accomplished through communication and matchmaking between $\mathrm{CHCs}$ and researchers at OCHIN; technology transfer and research methods tailored to community practice settings at Fenway Health; and infrastructure for a community IRB to provide community oversight of and priority setting for research at AAPCHO.

PBRNs can practice community-based strategies and act as bridges between academic and community practice settings. CHARN nodes were mindful of building capacity at member clinics, integrating member feedback and comments over several years. CHARN nodes were fortunate to have funding and time to build on the foundation that each node had already begun. We continue to strive toward a more balanced partnership.

A large network provides many benefits: (1) much-needed financial resources; (2) economic scale; (3) large sample sizes, enabling research on less common outcomes; (4) access to technological, administrative, and scientific resources and expertise via the data coordinating center and the academic affiliates which provides for a platform to share informa- 
tion and resources; and (5) rich diversity of member experiences and backgrounds. CHARN has helped each node and member CHC develop its research capacity individually and build the overall network capacity as well. In its first 2 years, CHARN experienced several challenges, such as long planning and start-up time to get policies, procedures, and governance structure in place, difficulties in obtaining additional resources to support such a large network, and a lengthy approval process to obtain buy-in from community partners. Despite these challenges, CHARN network members are successfully building tangible and sustainable research infrastructure at $\mathrm{CHCs}$ that is primarily due to the role of academic-community relationships in the capacity building process.

The authors gratefully acknowledge the CHARN health centers and research node centers for contributing the case studies and data used in preparation of this article. The authors are also grateful to Ms. LeNeva Spires, Publications Manager, Department of Family Medicine, OHSU, Portland, OR, for editing and publication assistance.

\section{References}

1. Oneha MF, Proser M, Weir RC. Community health centers: why engage in research? Washington, DC: National Association of Community Health Centers; 2012.

2. Seifer SD, Shore N, Holmes SL. Developing and sustaining community-university partnerships for health research: infrastructure requirements. Seattle, WA: Community-Campus Partnerships for Health; 2003.

3. Devoe JE, Gold R, Spofford M, et al. Developing a network of community health centers with a common electronic health record: description of the Safety Net West Practice-based Research Network (SNW-PBRN). J Am Board Fam Med 2011;24:597604.

4. Shoultz J, Oneha MF, Magnussen L, et al. Finding solutions to challenges faced in community-based participatory research between academic and community organizations. J Interprof Care 2006;20: $133-44$.
5. Calmbach WL, Ryan JG, Baldwin LM, Knox L. Practice-based research networks (PBRNs): meeting the challenges of the future. J Am Board Fam Med 2012;25:572-6.

6. Westfall JM, Fagnan LJ, Handley M, et al. Practicebased research is community engagement. J Am Board Fam Med 2009;22:423-7.

7. Calleson DC, Jordan C, Seifer SD. Community-engaged scholarship: is faculty work in communities a true academic enterprise? Acad Med 2005;80:317-21.

8. Seifer SD. Building and sustaining community-institutional partnerships for prevention research: findings from a national collaborative. J Urban Health 2006;83:989-1003.

9. Seifer SD, Gottlieb B. Transformation through partnerships. Prog Community Health Partnersh 2010;4:1-3.

10. Seifer SD, Krauel P. Toward a policy agenda for community-campus partnerships. Educ Health (Abingdon) 2001;14:156-62.

11. DeVoe JE, Weir RC, Rachman F, et al. Community Health Applied Research Network (CHARN): PBRN infrastructure and support. AHRQ PBRN Conference. Bethesda, MD, June 24, 2011.

12. National Association of Community Health Centers. America's health centers. Washington DC: NACHC; 2008.

13. Oneha MF, Beckham S. Re-examining community based research protocols. Pac Health Dialog 2004; 11:102-6.

14. DeVoe JE, Likumahuwa S, Eiff MP, et al. Lessons learned and challenges ahead: report from the OCHIN Safety Net West practice-based research network (PBRN). J Am Board Fam Med 2012;25: 560-4.

15. Song H. Developing patient-centered outcomes research projects in the community for the community-lessons learned from PACE CHARN research training. CHARN Steering Committee Meeting. Boston, MA, October 11, 2012.

16. Association of Asian Pacific Community Health Organizations. Fact sheet: developing an AAPCHO institutional review board. Oakland, CA: AAPCHO; 2012.

17. Proser M, Jester M, Shin P, et al. Federally qualified health center research capacity: enhancing community research engagement. Academy Health Annual Research Meeting, Orlando, FL, June 24, 2012. 